\title{
Throughput Improvement of IEEE 802.11 using Adaptive Slot Size
}

\author{
Sumeet Mundra $^{\dagger}$, and Vinay J. Ribeiro ${ }^{\S}$ \\ ${ }^{\dagger}$ Department of Electrical Engineering, ${ }^{\S}$ Department of Computer Science and Engineering \\ Indian Institute of Technology Delhi, Hauz Khas, New Delhi, India 110016 \\ Email: sumitmundra15@gmail.com, vinay@cse.iitd.ernet.in
}

\begin{abstract}
Although recent IEEE 802.11 wireless LAN standards (such as 802.11n/ac) support physical layer (PHY) transmission speed more than 10 times that of $\mathbf{8 0 2 . 1 1 g}$, the user level throughput has not seen a proportionate increase. The primary reason for this anomaly are the various overheads of channel access, preamble and acknowledgements.

In this paper, we present a MAC protocol that enables the access point (AP) in an infrastructure based 802.11 WLAN system to dynamically reduce the slot time used in the protocol thereby reducing the channel access overhead. A major component of slot time is the clear channel assessment (CCA) time, which is the time taken by a node to determine if the wireless medium is idle or busy. A key fact we exploit is that any node requires less time for CCA if the SNR of all nodes in the network at this node are high. Our protocol first determines the worst-case pair-wise SNR of all nodes in the WLAN by polling nodes by leveraging the PCF mode of 802.11 and then determines the optimal CCA and slot time. We present a theoretical analysis to determine the optimal CCA time given SNR and evaluate our protocol using the Qualnet Simulator.
\end{abstract}

\section{INTRODUCTION}

With advancement in MIMO technology, the IEEE 802.11ac standard is capable of transmitting data at a maximum rate of $1 \mathrm{Gbps}$ which is more than 20 times that of $802.11 \mathrm{~g}$ [1]. However, there has not been a proportionate increase in user experienced data rates. A primary reason behind this anomaly is the channel access overhead that is associated with a typical frame transmission in IEEE 802.11 based WLAN. Assuming a single stage back-off with default parameters, the average channel access overhead is given by $16 \mu \mathrm{s}+9.5^{*}$ slottime. Provided that slots are of duration $9 \mu \mathrm{s}$ as in $802.11 \mathrm{a} / \mathrm{n}$ [2], the average channel access delay is $101.5 \mu \mathrm{s}$. Thus, the channel access overhead alone is over $450 \%$ of the time required for transmitting a 1500 byte packet at 540 Mbps. In addition, there are overheads due to the physical layer preamble and MAC layer acknowledgements that further degrade the efficiency.

The slot time duration in 802.11 plays a key role in determination of channel access overhead. The $9 \mu$ s slot time in 802.11 is designed to include $4 \mu \mathrm{s}$ required for packet detection/clear channel assessment (CCA) [3] and 5 $\mu$ s known as turnaround time cumulatively for MAC layer processing, radio propagation delay and time required to switch the radio circuitry from receive to transmit mode. Thus, in order to reduce the slot time duration, either CCA time or turnaround time needs to be reduced.

The packet detection time in 802.11 depends on the signal to noise ratio (SNR) at the sensing node [4]. Hence, the stronger the received carrier, the faster it can be sensed. Thus, while 802.11 standard recommends $4 \mu \mathrm{s}$ as reliable time to sense carrier from farthest nodes in the network [2], it can be reduced significantly if the received signal strength at nodes is high, thereby providing an opportunity to shorten the slot time and channel access overhead. Based on this observation, we develop a modified MAC protocol that enables the access point (AP) to dynamically reduce the slot time duration to an optimal value based on SNR values at different nodes in the network and thus improves system throughput.

Consider a typical infrastructure based WLAN scenario as depicted in Figure 1 and Figure 2.

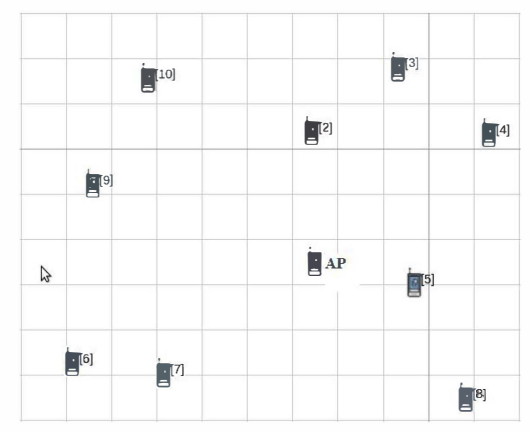

Fig. 1. Nodes take more time to perform CCA due to relatively low SNR

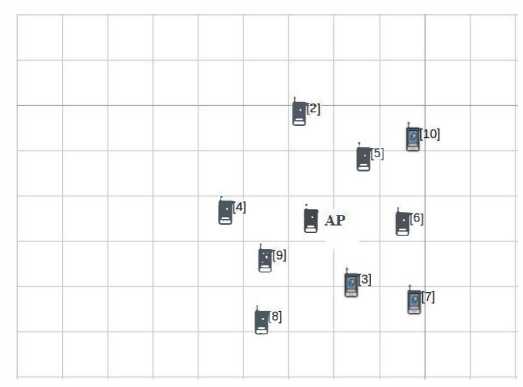

Fig. 2. Nodes take less time to perform CCA due to relatively high SNR

As the nodes are close to each other in the second case, each transmission results in a high SNR value observed at sensing nodes. Thus, nodes can perform CCA faster and hence require smaller slot times than in the first case.

In order to develop our adaptive slot MAC protocol, there are several challenges which we address. 
1) Determine the theoretical relation between the SNR value and the slot time duration: Intuitively, larger SNRs correspond to lower slot time durations. We perform a detailed study of the signal detection problem using the preamble structure in IEEE 802.11. We consider performance parameters such as the probability of detection and false alarm to determine the minimum slot time duration that allows even the farthest nodes in the network to reliably perform packet detection.

2) Procedure for WLAN to determine the smallest SNR value heard by any node in the network: The worst-case SNR determines the slot time to be used by the WLAN. To determine the worst SNR value, each node in the network needs to know SNR value due to transmission from every other node in the network. We thus require every node to transmit a packet at least once during every measurement period. The measurement period must be short enough to ensure that changes in SNR values over time due to nomadic mobility of users are tracked. At the same time, this period must not be too short, lest the measurement overhead becomes excessive.

3) Backward compatibility with the existing 802.11 protocols: Our protocol must not make radical changes to the PHY or MAC layers of the existing 802.11 protocols in order to be of practical use.

4) Allowing new nodes entry: It may so happen that a new node wishes to join the WLAN and the slot time currently used by the WLAN is too small for some other node to effectively detect its signal. This may unnecessarily cause collisions of its packets and lead to a delay of its joining the network.

5) Evaluation and validation of protocol: Another challenge is to implement and compare the proposed adaptive slot time protocol with the standard 802.11 protocol.

The main contributions of this paper are:

- The design of a protocol that improves the throughput of WiFi by adaptively reducing the slot time duration by taking into account the pair-wise SNR between nodes in the WLAN network.

- Determination of a theoretical relation between the optimal value of slot time duration for a given worstcase pairwise SNR in the network.

- Development of a simulation model in Qualnet of the proposed protocol for evaluation purposes and demonstrate its efficiency.

\section{RELATED WORK}

There is extensive literature available that deals with performance optimization of IEEE 802.11 based WLAN systems. Here we shall mention those which are most relevant to our work.

Eugenio Magistretti et al. [4] proposed a system that uses slots as small as $800 \mathrm{~ns}$ instead of conventional $9 \mu$ s slots to improve the throughput. Reduction in slot time duration is achieved through speculative transmission of the preamble by different nodes while the nodes contend for access to the medium. The nodes abort their transmissions as soon as they detect the channel to be busy thus allowing only the node with earliest transmission time to transmit. Though the idea is novel and about $100 \%$ gain is achieved, implementing this method requires significant changes in hardware and the 802.11 protocol. Our approach, in contrast, requires only slight modifications to the existing protocol without requiring any change in the hardware.

Frederico et al. [5] proposed a dynamic tuning of the exponential backoff algorithm and contention window parameter in the DCF mode operation. In our work, we focused on reducing the slot time duration itself instead of tuning the contention window parameter.

In [6] a novel preamble design was proposed that aims at reducing the PHY overheads by transmitting data in the preamble part itself and hence making more efficient utilization of bandwidth. This method attempts to reduce the preamble overhead, unlike ours which reduces CCA overhead.

\section{BACKGROUND PRELIMINARIES}

In this section, we present the background material for our present work by analysing the sequence of events associated with a typical packet transmission over the wireless medium, as shown in Figure 3 using the DCF channel access mechanism. The packet size is assumed to be 1500 bytes and that packet is transmitted using $802.11 \mathrm{n}$ at a $540 \mathrm{Mbps}$ data rate in $22 \mu \mathrm{s}$.

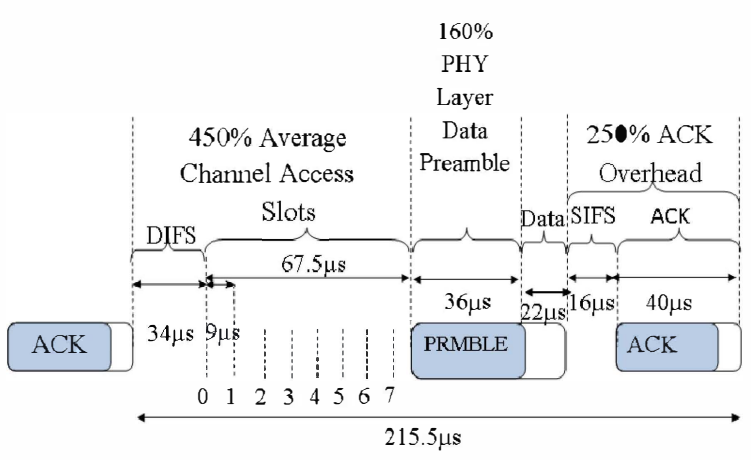

Fig. 3. Overheads in 802.11 at 540 Mbps

\section{A. Overheads}

The various overheads in 802.11 fall into the following categories.

Channel Access Overhead: The IEEE 802.11 standard mandates every node wishing to transmit a frame to sense the medium to be idle for a duration of DIFS (Distributed Inter Frame Space) which is $34 \mu$ s long and comprises of one SIFS (Short Inter Frame Space) $(16 \mu \mathrm{s})$ and 2 slots $(9 \mu \mathrm{s})$ each. The sending node then performs a random backoff by deferring its transmission for a random number of slots varying from 0 to CW-1 (contention window size). This is to allow a fair channel access to all the contending nodes in the network. Assuming a single stage backoff where $\mathrm{CW}$ remains at its minimum value $\mathrm{CW}_{\min }$ which is 16 for 802.11 [4], the average backoff is 


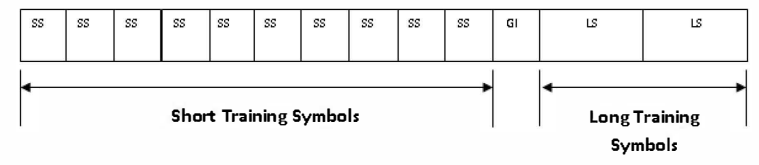

Fig. 4. Basic Format of 802.11 preamble

given by 7.5 slots. Thus, the average channel access overhead is given by $16 \mu \mathrm{s}+9.5^{*}$ slottime i.e., $101.5 \mu \mathrm{s}$. At $540 \mathrm{Mbps}$ data rate, this amounts to $450 \%$ of the actual data transmission time.

Preamble Overhead: Every packet transmission in 802.11 is preceded by a physical layer preamble and header. The function of preamble is important as it first enables the receiver to detect a packet transmission through correlation and identify different fields of the packet using time and frequency synchronization. Second, it also performs another important function of channel estimation required for data extraction by the receiver. Thus, $802.11 \mathrm{a} / \mathrm{g}$ consists of a preamble size of duration $20 \mu$ s (inclusive of PHY header) [4] and IEEE 802.11n operating in high throughput mode using four spatial streams has a maximum preamble of duration of $40 \mu \mathrm{s}$ [2] which is about $180 \%$ of the data transmission time.

Acknowledgement Overhead: On successfully receiving a frame, the receiver responds with an acknowledgement frame to the sender to indicate successful reception. The MAC layer at the receiver node requires time to process the incoming data and switch its radio from receive to transmit mode. Nodes are required to wait for an $\operatorname{SIFS}(16 \mu \mathrm{s})$ duration to perform all these tasks before transmitting an acknowledgement frame. This delay and time required for transmitting an ACK frame add to $56 \mu$ s of overhead in $802.11 \mathrm{n}$ at $540 \mathrm{Mbps}$, which equals $250 \%$ of the data transmission duration.

Till now, we have talked of overheads in connection with a single active node in the network. When there are multiple nodes contending for the medium, collision of frames may occur causing collision overhead.

Collision Overhead: As stated previously, each node desirous of transmitting a packet has to wait for a random backoff period determined by the value of their backoff counter before initiating its transmission. When there are multiple nodes competing for access to the medium, the probability of the backoff counter for two or more nodes expiring at the same slot increases causing packet collision. This results in collision overhead which is sum of channel access time, preamble time and data transmission time.

\section{Components of Slot Time}

Next, we provide an insight into the components of the slot time in 802.11. Slots in 802.11 consist of following time components - time required for packet detection, $\mathrm{Rx}-\mathrm{Tx}$ switching time, propagation delay and MAC processing delay [2].

Clear Channel Assessment: Also known as carrier sensing time, it is the time required by a node to perform packet detection [4]. Falsely detecting a transmission when there is none causes an unnecessary backoff at the transmitting node while missing legit transmissions may lead to collisions,

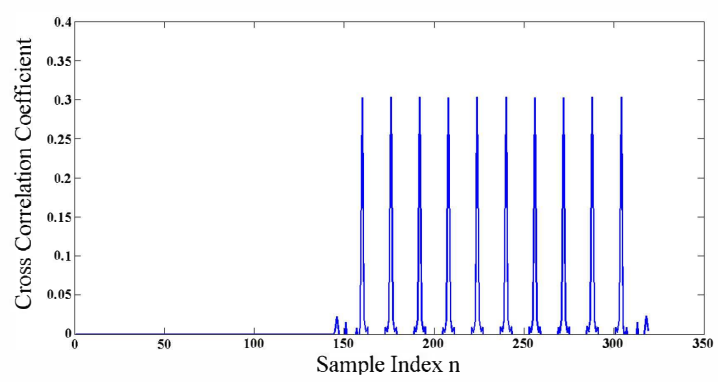

Fig. 5. Cross Correlation of IEEE 802.11a preamble with one Short Training Sequence

resulting in throughput loss in both the cases. In order to enable reliable detection, 802.11 standard mandates transmission of a preamble at the beginning of a frame.

The initial part of the preamble consists of ten identical sequences of Short Training Symbols (STS) of length 16 samples each and duration $0.8 \mu$ s obtained by OFDM modulation of known PN sequences [7]. The basic preamble format for 802.11 is depicted in Figure 4. A receiver detects an ongoing transmission by searching for the preamble in the received signal using energy detection [8] with autocorrelation or cross correlation techniques [9]. The autocorrelation function exploits the periodicity of the short training sequence by correlating the received signal with its conjugate delayed by a time period of one STS sequence. The autocorrelation function gives a peak when there is a match between the two sequences indicating the presence of the preamble. Figure 5 shows cross correlation of a single Short Training Symbol(STS) with one full preamble of IEEE 802.11a. We note that after an initial sequence of zeroes, ten peaks are obtained corresponding to ten repetitions of Short Training sequences in the preamble.

The reliability of detection is determined by the value of the normalized correlation coefficient. A packet is said to be detected if the value of the correlation coefficient exceeds the chosen threshold. The value of the correlation coefficient depends on the strength of the received signal and length of the preamble [4] and is given by:

$$
c_{n}=R S S * \Delta,
$$

where $c_{n}$ is the normalized correlation coefficient, RSS is received signal strength and $\Delta$ is gain of the preamble sequence. The preamble gain $\Delta$ is a linear function of length $L$ of the preamble sequence. Thus, for a given detection threshold, the length of the preamble sequence or the time required to detect an ongoing transmission depends on the signal to noise ratio (SNR) at the receiver. This is conservatively specified to be $4 \mu$ s by the 802.11 standard.

Rx-Tx switching time: Components such as antenna and RF frequency oscillator are common between transmitter and receiver circuitry and time is required to switch the radio circuitry from receive to transmit mode because of these shared components. 802.11 specifies $600 \mathrm{~ns}$ as the nominal switching time.

Propagation Delay: Electromagnetic waves travel with a finite speed and this results in a propagation delay. Thus, to 
compensate for this propagation delay, 802.11 includes $800 \mathrm{~ns}$ in the slot time duration.

MAC processing Delay: Every transmission from physical layer (PHY) is processed by the MAC layer and the MAC layer responds with appropriate commands back to PHY layer. This time depends on vendor specific implementation. The sum of the above three delays add up to $5 \mu$ s giving a total slot time duration of $9 \mu \mathrm{s}$.

\section{Optimal Slot Time and Performance IMPROVEMENT}

In this section, we determine the relation between SNR value and corresponding slot time duration that shall be used for computation of optimal slot time in our proposed protocol followed by a theoretical analysis of the resulting improvement in system efficiency.

We begin with determination of relation between slot time and SNR in this section. As described in previous section, the slot time duration in 802.11 is the sum of CCA time and other time delay components. Further, we also pointed out that CCA time depends on the signal strength of the received transmission. Thus, while trying to reduce the CCA time and hence the slot time based on observed SNR values, we must ensure that the new calculated value of slot time is optimal. Choosing a slot time duration that is unnecessarily long will reduce system throughput while choosing a slot time duration that is too short will lead to missed detections resulting in increased collisions. While 802.11 standard recommends about $4 \mu \mathrm{s}$ as reliable time to sense transmissions from far away nodes in the network, we determine a quantitative relation between the CCA time and SNR value to calculate an optimal value of slot time duration. To achieve this, we shall consider the decision statistics for the autocorrelation method used for preamble detection by an 802.11 receiver. For the autocorrelation technique, the normalized decision variable is defined as [10]:

$$
m_{n}=\frac{\left|c_{n}\right|^{2}}{\left(p_{n}\right)^{2}}
$$

where,

$$
c_{n}=\sum_{k=0}^{d-1} \sum_{m=0}^{L-1} r_{n+m+k L}^{*} r_{n+m+(k+1) L}
$$

and,

$$
p_{n}=\sum_{k=0}^{d-1} \sum_{m=0}^{L-1}\left|r_{n+m+(k+1) L}\right|^{2}
$$

where, $r_{n}$ represents samples of the incoming Short Training Sequence, $L$ is the length of each short training sequence which is 16 samples, and $d$ is the number of short training symbols taken into account to calculate the decision variable.

The value of this decision variable is compared against a threshold, $x_{T}$, to determine if a packet is present or not. Thus, a packet is said to be detected if the value of the decision variable exceeds the threshold while packet is said to be absent for cases where value of decision threshold is below the threshold.

For the noise only case, the pdf of the decision variable can be approximated as

$$
m_{n}=\frac{1}{2 d L} \chi_{2}^{2}
$$

where, $\chi_{2}^{2}$ is a chi-square distribution. For the noise only case, the mean and variance of the decision variable are given by

$$
E\left[m_{n}\right]=\frac{1}{d L}
$$

and

$$
\operatorname{Var}\left[m_{n}\right]=\frac{1}{d^{2} L^{2}}
$$

Similarly, for the signal with noise case, the decision variable can be approximated as a Gaussian random variable with mean and variance given by

$$
\mu_{m_{n}}=\frac{S N R^{2}}{(1+S N R)^{2}}
$$

and

$$
\operatorname{Var}\left[m_{n}\right]=\frac{2 S N R^{2}\left[\left(1+\mu_{m_{n}}\right) S N R+\left(1+2 \mu_{m_{n}}\right)\right]}{d L(1+S N R)^{4}}
$$

We next find expressions for Probability of detection $P_{D}$ and Probability of false alarm $P_{F A}$. Probability of detection $P_{D}$ is the probability of a packet being detected when there is actually one and probability of false alarm $P_{F A}$ is the probability of detecting a packet when there is none. The expressions for $P_{D}$ and $P_{F A}$ are as described below:

$$
\begin{gathered}
P_{D}=\int_{x_{T}}^{\infty} f_{1}(x) d x \\
P_{F A}=\int_{x_{T}}^{\infty} f_{2}(x) d x
\end{gathered}
$$

Here, $f_{1}(x)$ and $f_{2}(x)$ are the pdfs of the decision statistic variable for the cases where a signal is present and where only noise is present respectively.

We use the above mentioned expressions and distributions to evaluate the two probabilities. Finally we determine the relation between SNR and number of STS symbols required for packet detection, $d$, by equating the detection thresholds for two probabilities and limiting the probability of false alarm $P_{F A}$ to $10^{-8}$ and probability of detection to $1-10^{-8}$.

We must notice that each value of $d$ corresponds to one Short Training Symbol which is of duration $0.8 \mu \mathrm{s}$. Thus, a value of $d$ equal to 5 corresponds to a slot time of $9 \mu$ s duration and we decrease the value of slot duration by $0.8 \mu$ s every time value of $d$ decreased by 1 . Further, as we are assuming autocorrelation technique being used for signal detection, we require at least two Short Training symbols for packet detection.

Figure 6 depicts the calculated value of slot time for different values of SNR assuming probability of false alarm $P_{F A}$ as $10^{-8}$ and probability of detection as $1-10^{-8}$ as stated earlier.

Performance Improvement: Next, we determine the improvement in data efficiency resulting from reduction in slot time at different transmission data rates under different conditions. We define data efficiency as the percentage of total time used for actual data transmission time. We determine an expression for data efficiency in 802.11 assuming DCF 


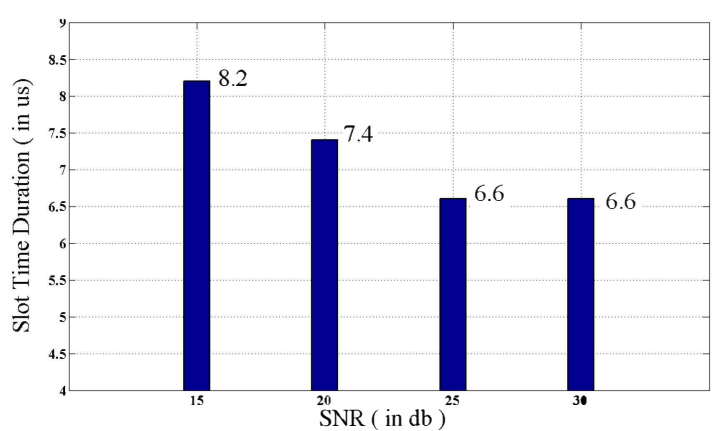

Fig. 6. Slot Time at different values of SNR

TABLE I. PARAmeter VAlues used in TheOretical ANalysis

\begin{tabular}{|l|l|}
\hline Parameter Name & Value \\
\hline Packet Size & 1500 bytes \\
\hline SIFS & $16 \mu \mathrm{S}$ \\
\hline DIFS & SIFS $+2 *$ slottime \\
\hline$C W_{\text {min }}$ & 15 \\
\hline PHY-Preamble Duration & $36 \mu \mathrm{s}$ \\
\hline ACK Frame Duration & $4 \mu \mathrm{s}$ \\
\hline PHY-Data Rate(Mbps) & $54,135,216,540$ \\
\hline
\end{tabular}

channel access mechanism. For this, we consider various time components involved in transmission of a packet in a WLAN. As already described in Section III, the total time duration required for transmission of a data packet can be represented as

$$
\begin{aligned}
T_{\text {Total }} & =T_{\text {DIFS }}+T_{\text {backoff }}+T_{\text {Preamble }} \\
& +T_{\text {data }}+T_{\text {SIFS }}+T_{\text {Preamble }}+T_{A C K}
\end{aligned}
$$

We can then define Data efficiency as :

$$
\text { average data efficiency }=\frac{T_{\text {data }}}{T_{\text {total }}} * 100 \%
$$

For simplicity, we have assumed a single stage backoff for which on average

$$
T_{\text {backoff }}=\frac{C W_{\min }}{2}
$$

where $C W_{\min }$ is 15 and that RTS/CTS feature is turned off. Table I lists the values of different parameters used in calculations.

Figure 7 shows the different overheads at various data rates assuming a slot time of $9 \mu \mathrm{s}$. Here, $T_{\text {channel }}$ is the channel access delay and is the sum of $T_{D I F S}$ and $T_{\text {backoff }}$ parameters as defined in equation 8. Similarly, $T_{a c k}$ is the sum of $T_{S I F S}, T_{\text {Preamble }}$ and $T_{A C K}$. We notice from the figure that as data rate increases, data transmission time $T_{\text {data }}$ decreases and hence the data efficiency reduces from around $53 \%$ at 54 Mbps to about $10 \%$ at 540 Mbps while the channel access overhead increases from $24 \%$ to $47 \%$. Similary, Figure 8 depicts overheads at various overheads for slot time durations of $6.6 \mu \mathrm{s}$.

Table II shows the data efficiency and $\%$ gain at different data rates for sending a packet of size 1500 bytes (maximum sized datagram) for the two extreme cases, namely, first when the slot time is $9 \mu \mathrm{s}$ and second when the slot time is $6.6 \mu \mathrm{s}$. We notice that Table II shows the maximum possible improvement for reducing slot time to $6.6 \mu \mathrm{s}$. In reality, however, the gains

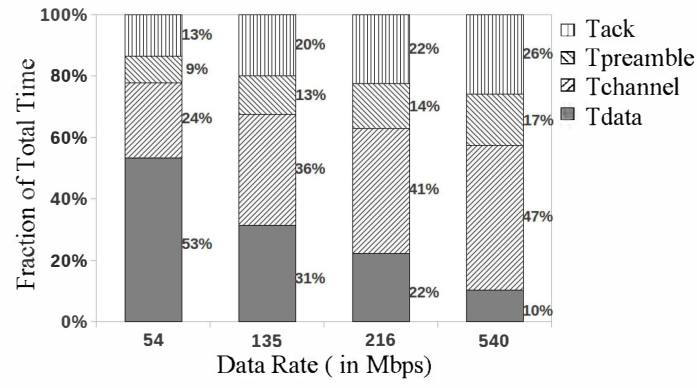

Fig. 7. Overheads in 802.11 at different data rates for $9 \mu$ s slots

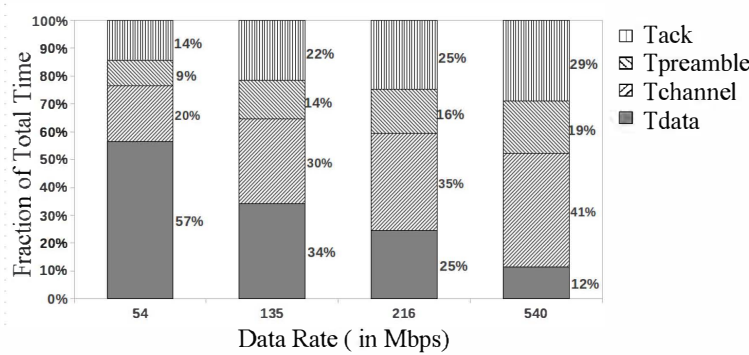

Fig. 8. Overheads in 802.11 at different data rates for $6.6 \mu$ s slots

achieved would be lower as slot time duration won't always be $6.6 \mu$ s but a value that changes depending on the observed SNR values at different nodes. Further, we have assumed a backoff duration of $\frac{C W_{\min }}{2}$ on average. Though this is true for a single node transmission, the average backoff duration reduces with increase in number of contending nodes thereby reducing the impact of channel access overhead on system performance.

TABLE II. DATA EFFICIENCIES AND \% GAIN FOR A SINGLE NODE SENDING 1500 BYTE PACKETS FOR SLOTS $9 \mu$ S AND $6.6 \mu \mathrm{S}$

\begin{tabular}{|c|l|l|c|}
\hline Data Rate [Mbps] & $\begin{array}{l}\text { Efficiency (\%) } \\
\text { for slot time } \\
9 \mu \mathrm{s}\end{array}$ & $\begin{array}{l}\text { Efficiency (\%) } \\
\text { for slot time } \\
6.6 \mu \mathrm{s}\end{array}$ & Gain (\%) \\
\hline 54 & 57.9 & 61.57 & 6.34 \\
\hline 135 & 31.48 & 34.24 & 8.77 \\
\hline 216 & 22.31 & 24.55 & 10.06 \\
\hline 540 & 10.3 & 11.52 & 11.83 \\
\hline
\end{tabular}

Figure 9 and 10 compares the overheads and efficiency at different slot time values respectively. These results are corresponding to a data rate of $540 \mathrm{Mbps}$ and for a single active transmitter with packet size set to 1500 bytes. Note that as slot time reduces from $9 \mu \mathrm{s}$ to $5.8 \mu \mathrm{s}$, the fraction of data transmission time increases from $10 \%$ to $12 \%$, a gain of about $20 \%$ in throughput.

Although in this paper we focus on reducing the CCA component of the slot time duration, we compute the gains that can be achieved if the MAC processing delay which is the other major delay component of the slot time is eliminated. In Figure 11, we depict various overheads by reducing the MAC processing delay to zero in addition to reducing the CCA time component to $1.6 \mu \mathrm{s}$. This results in a slot time duration of $2.6 \mu \mathrm{s}$. Observe that the data efficiency increases from 54\% to $63 \%$ at $54 \mathrm{Mbps}$ and from $10 \%$ to $14 \%$ at $540 \mathrm{Mbps}$. This gives improvement in throughput of $16.67 \%, 29 \%, 36 \%$ and $40 \%$ at 54, 135, 216 and 540 Mbps respectively. 


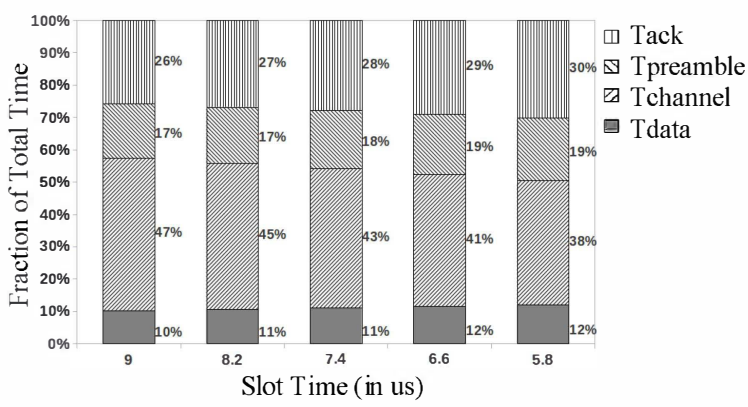

Fig. 9. Overheads in 802.11 at different slot time values for data rate 540 Mbps

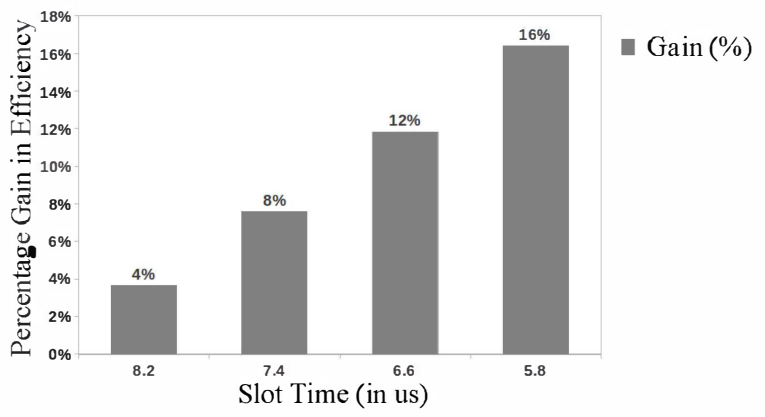

Fig. 10. Percentage gain in efficiency at different slot time values at data rate of 540 Mbps compared to a $9 \mu$ s slot time.

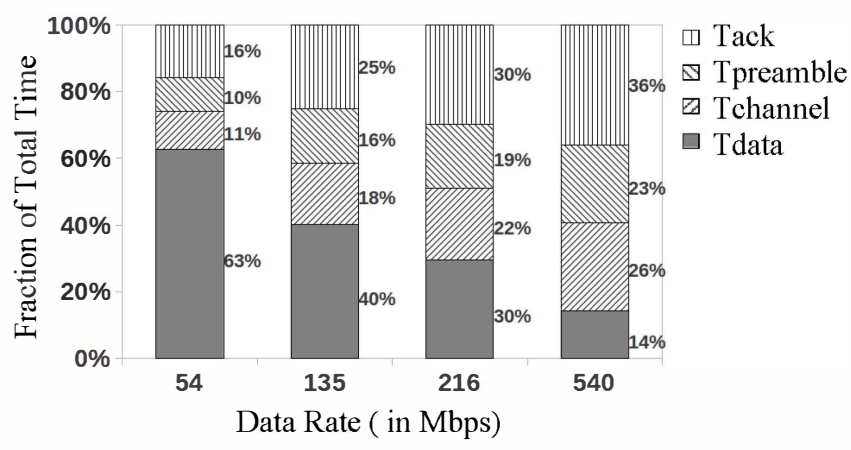

Fig. 11. Overheads by reducing slot time duration to $2.6 \mu \mathrm{s}$

\section{PROPOSED PROTOCOL}

As mentioned in Section I, the access point must consider the worst SNR values while calculating the slot time duration to ensure that the new calculated value of slot time duration is long enough for each node in the network to perform carrier sensing reliably.To determine the worst SNR, each node must know the SNR due to transmission from every other node in the network which requires each node to transmit at least once before a slot time computation can be made. Our protocol must provide a solution to this issue. Secondly, nodes in a wireless network are mobile and their positions may change while they are connected to the network. This would result in a change in SNR values observed at different nodes. Our proposed solution must ensure that every time a slot time value is computed, it corresponds to the most recent SNR values. Next, our proposed protocol enables the nodes in the network to exchange packets using the standard Distributed coordination function (DCF) channel access mechanism but

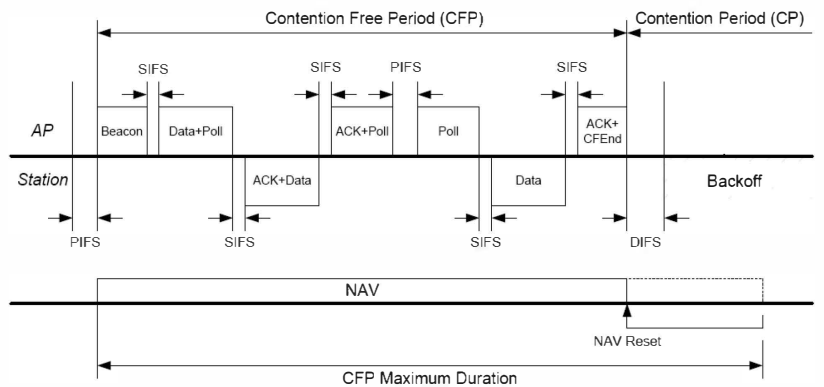

Fig. 12. Frame Transfer during PCF.

with a smaller slot time duration. Now, this may impose a problem for a new node who wishes to join the network. Due to smaller slot time duration (implying SNR values above a certain threshold), the nodes who are already joined to the network may not be able to hear the association requests from nodes trying to associate with the network due to weak SNR values resulting in collision. Thus, the association requests may never be received by the AP causing the new nodes to scan alternative channels or look for other access points for connectivity. Our protocol must allow for a new node to join the network without any problems. In the next section, we explain our protocol while we address each of the above mentioned challenges.

As our solution requires each node to transmit at least once, we intend to ensure this using the Point Coordination function (PCF) mode with the AP acting as Point coordinator(PC). PCF [11] is an optional access mechanism which provides contention free frame transfer using a poll and response technique. During PCF, the Point Coordinator(PC), which usually is the AP, controls access to the channel and determines which node will transmit next. During this contention free period, the PC delivers data to nodes while at the same time polling nodes on its polling list. In the context of our protocol, we wish to poll all the nodes currently associated with the AP. On receiving a poll, a node must respond either with a data or null data frame depending on whether or not the node has pending data. Polling nodes in this way would ensure that each node has transmitted at least once and each node knows the worst SNR due to transmission from all other nodes in the network not hidden from it. We address the case of hidden nodes subsequently. We call this period as the measurement period as SNR measurements are performed during this interval. Figure 12 depicts the basic frame transfer procedure during PCF.

Various steps that shall be performed for optimal slot time calculation are as listed below:

- $\quad$ The AP indicates the start of the measurement period by entering into PCF mode and broadcasting a beacon frame in the network.

- The AP then selects and polls a group of nodes from the polling list. On receiving a POLL frame, a node responds back to the AP either by sending a DATA + POLL-ACK (acknowledgement) or POLL-ACK frame depending on whether node has any data to send or not. 
- As the medium is shared, the response from the polled node is overheard by all other nodes in the network. At each of these listening nodes, the SNR value resulting from this transmission from the polled node is measured and stored. Nodes which are hidden from the polled node will not hear it and hence will not have this node among the list of nodes overheard.

- The AP continues to poll the next node in the group until all the nodes in the group are polled once. At each transmission from the polled node, the stored SNR value at the remaining nodes is updated to the new value if this new value is smaller than the previous stored minimum SNR value. This ensures that the stored SNR value at the node corresponds to the worst SNR value from all overheard nodes.

- The above cycle repeats with AP selecting a new set of nodes to be polled until all nodes in the network are polled.

- $\quad$ By the end of this first round of polling, each node in the network has transmitted atleast once and each node knows the worst SNR at its end due to transmission from other overheard nodes in the network.

- The AP now begins a second and final round of polling. This time, however, in response to the POLL frame from AP, individual nodes respond back with the stored minimum SNR values and corresponding MAC addresses of overheard nodes.

- At the end of this second and final polling round, the AP knows the measured SNR values at all the nodes and uses the least of these SNR values to compute the new value of slot time. In case any node does not report the MAC address of some other node in the network, the AP assumes that they are hidden from each other and hence uses the default slot time of 802.11. This ensures that the computed slot time durtion is optimal and allows reliable packet detection by every node in the network. The AP then broadcasts this value to the nodes in the network.

The above mentioned steps are repeated periodically by the AP. Hence, the slot time duration is updated periodically before it becomes stale and corresponds to the current network configuration, i.e., relative position of nodes with respect to each other. This way we take into account the possibility of node mobility.

In a typical WLAN, the access point transmits beacon frames at regular intervals to indicate their presence and to maintain the network. The client nodes listen and process beacon frames to gather information about the various access points. A typical beacon frame consists of various fields, one of which is CF (Contention Free) parameter set information which is present in beacons generated by AP's supporting PCF mode. This CF field provides information regarding the polling period to different client nodes as well as other nodes wishing to connect to the network. Thus, every node listening to beacon frame knows when the next polling round would start. In our proposed protocol, at the start of every polling round, we reserve a time duration equal to the sum of the time required to transmit an association request from a client node to $\mathrm{AP}$ and an association response from AP to client node.

Further, we make a slight modification to the association procedure where we require each node trying to associate with the network to send the association request frame immediately after start of the polling period. Thus, this way we ensure that every new node is able to connect to the network without any difficulty.

\section{Simulation Results}

In this section we run simulations to evaluate the proposed protocol for improvement in system performance. We first explain the simulation settings used for performing the simulations and then analyze results obtained for comparison with the standard protocol.

\section{A. Simulation Settings}

The simulation model of the proposed protocol was implemented and evaluated using the Qualnet Simulator [12]. Our simulations were confined to a single infrastructure based WLAN where multiple nodes communicate with a single access point. In our simulations, nodes were placed randomly around the access point and random mobility was given to all nodes using random waypoint model in Qualnet except the access point to ensure that SNR values observed at nodes changed as the simulation progressed. No nodes were assumed to be in power save mode and thus each node was available to receive frames from the AP at all the times. Simulations were done using 802.11a and 802.11n data rates. Each of our simulation was run for a period of 30 s and results were averaged over 5 such executions. Table III gives the values of various configuration parameters used in simulations.

TABLE III. Simulation PARAMETERS

\begin{tabular}{|c|c|}
\hline Parameter & Value \\
\hline Physical Layer & $802.11 \mathrm{a} / \mathrm{n}$ \\
\hline Transmission Rate & Varied from 54 to 540 (Mbps) \\
\hline Transmission Power & $20 \mathrm{dBm}$ \\
\hline PHY Preamble+Header duration & $36 \mu \mathrm{s}$ \\
\hline SIFS Time & $16 \mu \mathrm{s}$ \\
\hline RTS/CTS Threshold & 2347 bytes \\
\hline$C W_{\min }$ & 15 \\
\hline$C W_{\operatorname{mex}}$ & 1031 \\
\hline Polling Repetition Interval & $800 \mathrm{TU}$ \\
\hline One Time Unit(TU) & $1024 \mu \mathrm{s}$ \\
\hline Number of Nodes & Varied from 10 to 30 \\
\hline Packet Size & 1500 bytes \\
\hline
\end{tabular}

\section{B. Simulation Results}

Figure 13 compares the number of data packets transmitted by a single transmitter at different data rates for our adaptive slot protocol and the 802.11 protocol. A random node is selected as transmitter for each simulation and the transmitting node is fully backlogged with data traffic with packet size 1500 bytes. We observe that as the data rate increases, the relative gain of the modified protocol over 802.11 increases from $4.29 \%$ at $54 \mathrm{Mbps}$ to $5.8 \%$ at $540 \mathrm{Mbps}$.

In our simulations, slot time duration varies from $9 \mu$ s to $6.6 \mu$ s depending on the position of nodes in the network and is not always $6.6 \mu \mathrm{s}$ and thus the gains are less than those 
shown in Table II. Further, the gains mentioned in table II were calculated assuming that 1500 byte packets were transmitted back to back. But, in our case, there are other overheads involved such as those due to beacon frames, other control packets, collision and polling overhead which reduces the throughput.

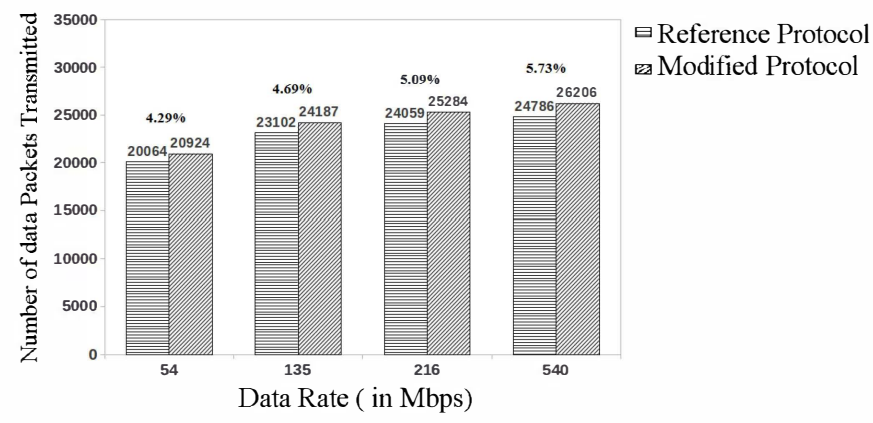

Fig. 13. Number of data packets transmitted at different data rates for single active transmitter

Figure 14 compares the number of data packets transmitted at different data rates for the two protocols but this time the number of active transmitters are 30 . As explained previously, the average backoff interval reduces with increase in number of contending nodes thereby reducing the average channel access overhead and lowering the impact of slot time duration. However, at the same time, polling overhead decreases with increase in number of active nodes in the network.

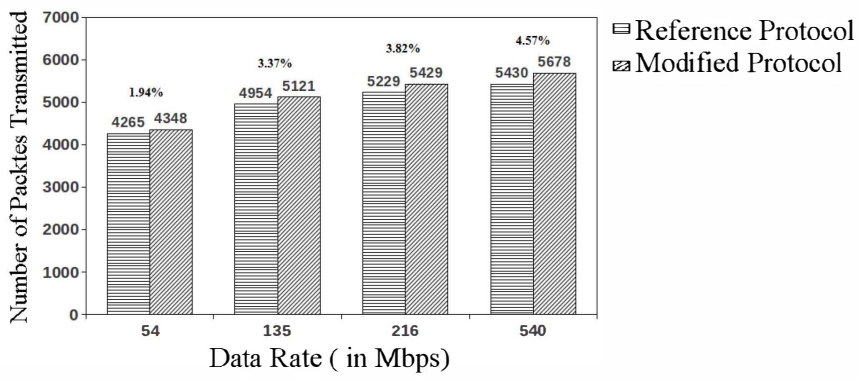

Fig. 14. Number of data packets transmitted at different data rates for 30 active transmitters

\section{CONCLUSION AND FUTURE WORK}

As the dynamic calculation of slot time duration required every node in the network to know the SNR value due to transmission from every other node in the network, we identified polling of different client nodes by AP as a procedure to ensure that each node transmitted at least once before slot time computation is made by the AP. Second, a quantitative relation between the SNR and slot time duration was derived keeping an appropriate threshold on performance parameters such as probability of detection and false alarm. We then presented a theoretical analysis of the improvement in system efficiency at different slot times and data rates. Finally, a simulation model of the proposed protocol was implemented using Qualnet Network Simulator, simulations were run and results obtained were analysed to see the improvement in system efficiency. We found that the gain achieved was maximum for 540 Mbps transmission rate and when there was a single active transmitter. However, as the number of active transmitters increased, the gain decreased owing to decrease in average backoff duration.

While our present work focused on reducing the CCA time, one of the components of slot duration in 802.11, reducing MAC processing delay, the other major component of slot time is still an area open for research and experimentation.

While our version of proposed protocol polls nodes in the network at fixed time interval, work could be done to develop a simulation model that allows the AP to dynamically adapt polling frequency based on mobility of nodes in the network thereby avoiding unnecessary polling overheads.

One could also focus on reducing the other overheads such as PHY preamble and MAC level acknowledgements and combine those techniques with the current work to obtain better results.

\section{REFERENCES}

[1] "802.11ac: The fifth generation of Wi-Fi," White Paper, Cisco, August 2012.

[2] "IEEE standard for information technology- local and metropolitan area networks- specific requirements- part 11: Wireless LAN medium access control (mac)and physical layer (phy) specifications amendment 5: Enhancements for higher throughput," IEEE Std 802.11n-2009 (Amendment to IEEE Std 802.11-2007 as amended by IEEE Std 802.11k2008, IEEE Std 802.11r-2008, IEEE Std 802.11y-2008, and IEEE Std 802.11w-2009), pp. 1-565, 2009.

[3] J. Fang, K. Tan, Y. Zhang, S. Chen, L. Shi, J. Zhang, Y. Zhang, and Z. Tan, "Fine-grained channel access in wireless lan," Networking, IEEE/ACM Transactions on, vol. 21, no. 3, pp. 772-787, 2013.

[4] E. Magistretti, K. K. Chintalapudi, B. Radunovic, and R. Ramjee, "WiFi-Nano: reclaiming wifi efficiency through $800 \mathrm{~ns}$ slots," in Proceedings of the 17th annual international conference on Mobile computing and networking, ser. MobiCom '11, 2011, pp. 37-48.

[5] F. Cali, M. Conti, and E. Gregori, "Dynamic tuning of the ieee 802.11 protocol to achieve a theoretical throughput limit," Networking, IEEE/ACM Transactions on, vol. 8, no. 6, pp. 785-799, 2000.

[6] Y. Wang, J. Oostveen, A. Filippi, and S. Wesemann, "A novel preamble scheme for packet-based OFDM WLAN," in Wireless Communications and Networking Conference, 2007. WCNC 2007. IEEE, 2007, pp. 14811485 .

[7] J. Heiskala and J. Terry, Ph.D., OFDM Wireless LANs: A Theoretical and Practical Guide. Indianapolis, IN, USA: Sams, 2001.

[8] I. Ramachandran and S. Roy, "Clear channel assessment in energyconstrained wideband wireless networks," Wireless Communications, IEEE, vol. 14, no. 3, pp. 70-78, 2007.

[9] K. Jamieson, B. Hull, A. Miu, and H. Balakrishnan, "Understanding the real-world performance of carrier sense," in Proceedings of the 2005 ACM SIGCOMM workshop on Experimental approaches to wireless network design and analysis, ser. E-WIND '05, 2005, pp. 52-57.

[10] T. Schmidl and D. Cox, "Robust frequency and timing synchronization for OFDM," Communications, IEEE Transactions on, vol. 45, no. 12, pp. 1613-1621, 1997.

[11] M. Ergen, "IEEE 802.11 tutorial," University of California Berkeley, Tech. Rep., June 2002.

[12] QualNet 5.2 Programmers Guide, Scalable Network Technologies Inc., Los Angeles, CA, 2011. [Online]. Available: https://www.scalablenetworks.com 